\title{
Comparative Study between Robomind and Scratch as Programming Assistance Tool in Improving Understanding of the Basic Programming Concepts
}

\author{
M. Faisal ${ }^{1}$, Rosihan Ari Yuana ${ }^{1}$ Basori $^{1}$ \\ ${ }^{1}$ Sebelas Maret University., Ir. Sutami Street, No.36A, Jebres, Surakarta, Central Java 57126, \\ Indonesia
}

\begin{abstract}
Dull learning, not prioritizing to the learning experience and process that is a teacher-centered learning becomes an obstacle in process of learning. The use of Programming Assistance Tool (PAT) can be a solution to overcome the obstacle. By using PAT, a teacher can create fun activities in the learning process by combining learning activities and playing. Robomind and Scratch are two of many PATs in the world. Both have different programming environments concepts, Robomind is a syntaxbased concept, while Scratch is a block-based concept with a drag-and-drop interface. The purpose of this research is to identify the difference of both PATs in improving understanding of decision making and looping. This research used the quasi-experiment method with nonequivalent control group design. The sampling technique used a random sample with total sampling type. The sample used 49 people who recently studied the basic concepts of programming (novice programmer) at the formal education level. Based on the analysis using the t-Test, it is known that there are differences in ability to improve the understanding of decision making and looping on both PATs. Meanwhile, by using the gain test, it was found that Scratch is slightly superior (low gain difference) than Robomind.
\end{abstract}

\section{Keywords: programming tools; scratch; robomind}

\section{INTRODUCTION}

Skilled human resources are one of the important aspects of improving the competitiveness of IT (information technology) industry software development. The low number of enthusiasts in the field of science and technology has produced a decrease in the number of software developers which resulted in the increased cost of software development [1]. Education can be a way to increase the number of skilled workers in the field of software development. Students need to be equipped with technological skills and knowledge for future success [2]. However, learning programming in the classroom is not considered as an easy thing by learners. Based on research of Yuana [3], 73\% stated that programming is one of the most difficult subjects to be faced in lectures, $96 \%$ stated that programming is very important to be studied by students in computer and information major, and 90\% stated that learning media is needed which makes it easier the understanding to support programming learning.

The main obstacles faced by learners occur when the process of programming learning in the early years when just starting to learn programming becomes a challenge 
that is very difficult to pass by the learners. But, when successfully mastered the ability to think systematically and logically, learners have been able to implement these capabilities in the form of computing in overcoming the problems [4]. Therefore, the early process of learning the basic concept of programming has a very important role. The student-centered learning could be an option because the method is designed to create a dynamic studying environment by actively participating and contributing during the learning process [5]. This is in line with flow theory, the human can do anything in the best possible way if they totally involved in a fun activity [6]. One of a way that could be used to create a fun activity in the learning process is to integrate learning activities with play.

PAT (Programming Assistance Tool) becomes one of the tools that can be used to support related learning [7]. The use of PAT is believed to motivate the learners due to the learning activities are done as if playing a game. A student who doesn't know programming before becomes motivated to study programming. This is corresponding to the result of this research which is done to the unknowing programming students. The result is $85 \%$ of student have understood better [8]. The PAT that can be used in programming learning is Robomind and Scratch. Implementation of the problem based leaning using Robomind could enhance students understanding of the programming algorithm [9]. The PAT rarely used as a tool to support programming learning activities and generally, learners are not aware of using it [7]. Educators and learners do not know how to use PAT effectively in support of learning programming concept [1]. The effectiveness from those both of PAT is important to be known by the educators in order to be used in accordance with the instructional design that will be applied by the educators in the classroom. Not to mention, the effectiveness of using a PAT is not necessarily the same with other PAT effectiveness. This is because the PAT is developed by different developers with a development concept that is not necessarily the same. This study aims to compare the effectiveness of both PATs, between Robomind and Scratch, in enhancing the understanding of the basic concept of programming. As well, to identify the differentiating aspects between Robomind and Scratch in enhancing the understanding of the basic concept of programming. The researches which are related to the comparison of Robomind and Scratch that have bren done are merely comparing the features. However, the researches which are related to the comparison of the effectiveness in classroom learning have not been done yet. At the end of this study will be addressed to answer the problem statement (research question) as follows:

$\mathrm{RQ}_{1}$ : Is there any differences in understanding of the basic concept of programming when using Robomind and when using Scratch?

$\mathrm{RQ}_{2}$ : Which one is more effective between Robomind and Scratch in improving understanding of the basic concept of programming?

$\mathrm{RQ}_{3}$ : What are the aspects that differentiate Robomind and Scratch in improving understanding of the basic concept of programming?

\section{METHODS}

This study uses quantitative research approach with the quasi-experimental method. This study uses nonequivalent control group design research. The population in this study amounted to 49 vocational high school students. The population is derived from 
the number of learners in each class who have followed the lesson using PAT for at least two meetings (three hours) in the learning session using PAT in the classroom, following the post-test, and are learners who just learn the basic concepts of programming (novice programmer) at formal education level. The meeting held eight times and the eighth meeting was post-test. Every single meeting spent 45 minutes. The first to the third meeting is a learning session using PAT. The fourth meeting was learning the introduction of the programming language by connecting it with the way PAT work has previously been studied. The fifth to the seventh meeting was a learning session using $\mathrm{C}++$ Programming Language. The discovery learning method used in the first and the third meeting, while problem-based learning method used in the second, third, fourth, fifth, sixth, and seventh meeting. The effectiveness of understanding the basic concept of programming that will be measured in this study refers to the measurement of indicators' achievement on basic competencies at C2 level (understanding) of Bloom's Taxonomy Revised edition by Krathwohl [10]. Understanding the basic concept of programming to be measured consists of understanding the concept of decision making and looping (see Table I).

TABLE I. INDICATORS AND BASIC COMPETENCIES FOR PROGRAMMING UNDERSTANDING[11]

\begin{tabular}{|cl|}
\hline $\begin{array}{c}\text { Standard Competencies } \\
\begin{array}{c}\text { Implementing a decision making } \\
\text { control structure in the } \\
\text { programming language }\end{array}\end{array}$ & 1. $\begin{array}{l}\text { describing the function of the use of the } \\
\text { decision making control structure } \\
\text { describing the syntactic function of the } \\
\text { decision making control structure }\end{array}$ \\
\hline $\begin{array}{c}\text { Implementing a looping control } \\
\text { structure in the programming } \\
\text { language }\end{array}$ & 1. $\begin{array}{l}\text { describing the function of the use of the } \\
\text { looping structure control } \\
\text { describing the syntactic function of } \\
\text { looping structure control }\end{array}$ \\
\hline
\end{tabular}

The sampling technique in this study used random sampling with total sampling type. So that the number of samples used is the same as the population. The sample divided into two groups, namely Robomind Class (got treated with PAT Robomind learning) with the number of participants as much as 21 people and Scratch Class (got treated with PAT Scratch learning) with the number of participants as much as 28 people. The data collection technique in this study uses test method. The test used is to measure understanding of the basic concept of programming that students have in accordance with the achievement indicators (see Table I). The procedure of this study consists of: (1) sample group equality test by pretest; (2) giving the treatment for both of sample group; (3) giving post-test to measure understanding the basic concept of programming; (4) analyzing the result. To answer the first and the second research question in this study, the prerequisite test used in this study consisted of t-test balance, normality test, and homogeneity test. Hypothesis test used by t-test and gain test. As well as using descriptive analysis to answer the third problem statement. 


\section{RESULT}

1. $R Q_{1}$ : Is there any difference in improvement of understanding the basic concept of programming when using Robomind and when using Scratch?

Based on the calculation of pretest results in the two groups of samples using the normality test, homogeneity test, t-test balance obtained the calculation results as in Table II and Table III. The t-test balance analysis performed on pretest results is to show the equality of both of sample groups. The analysis used balance t-test. This test was conducted while the two groups have not been treated yet and aimed at investigating whether both groups are balance [12]. This research only used the balance group which was based on the result of pre-test. Based on Table II it is known that the data on pretest value is normal. This is known from the Sig value Robomind Class in the Kolmogorov-Smirnov test of 0.075 and Sig value Scratch Class in the KolmogorovSmirnov test of 0.200 , the value is greater than the error rate (0.05). The Sig value in the Levene test is 0.080 . The value is greater than the error rate $(0.05)$, so it is known that pretest result is homogeneous. The Sig (2-tailed) value in the t-test balance is 0.282. The value is greater than the error rate $(0.05)$, so it is known that pretest result is balanced (no significant difference in the two experimental classes).

TABLE II. NORMALITY TEST IN PRETEST RESULTS

\begin{tabular}{|ccc|}
\hline & \multicolumn{2}{c|}{ Kolmogorov-Smirnov ${ }^{a}$ test } \\
\cline { 2 - 3 } & Statistic & Sig \\
\hline Robomind Class & 0,150 & 0,075 \\
Scratch Class & 0,106 & 0,200 \\
\hline
\end{tabular}

a. Lilliefors Significance Correction

TABLE III. HOMOGENEITY TEST, AND T-TEST BALANCE IN PRETEST RESULTS

\begin{tabular}{|ccccc|}
\hline & \multicolumn{3}{c}{ Levene test } & \multicolumn{3}{c|}{ T-test balance } \\
\cline { 2 - 5 } & $\mathrm{F}$ & $\mathrm{Sig}$ & $\mathrm{t}$ & $\begin{array}{c}\text { Sig. } \\
(2-\text {-tailed })\end{array}$ \\
\hline Value & 3,170 & 0,080 & 1,085 & 0,282 \\
\hline
\end{tabular}

The calculation of normality test, homogeneity test, t-test of post-test result can be seen in Table IV and Table V. T-test in post test result is used to answer research question number 1.

TABLE IV. NORMALITY TEST IN POST TEST RESULTS

\begin{tabular}{|ccc|}
\hline & \multicolumn{2}{c|}{ Kolmogorov-Smirnov ${ }^{a}$ test } \\
\cline { 2 - 3 } & Statistic & Sig \\
\hline Robomind Class & 0,174 & 0,095 \\
Scratch Class & 0,103 & 0,200 \\
\hline
\end{tabular}

a. Lilliefors Significance Correction 
TABLE V. HOMOGENEITY TEST, AND T-TEST BALANCE IN POST TEST RESULTS

\begin{tabular}{|ccccc|}
\hline & \multicolumn{3}{c}{ Levene test } & \multicolumn{2}{c|}{ T-test } \\
\cline { 2 - 5 } & $\mathrm{F}$ & $\mathrm{Sig}$ & $\mathrm{t}$ & $\begin{array}{c}\text { Sig. } \\
(2 \text {-tailed })\end{array}$ \\
\hline Value & 3,757 & 0,059 & $-2,030$ & 0,048 \\
\hline
\end{tabular}

Based on the calculation of post-test results in both of sample groups using normality test, homogeneity test, balance test $t$ obtained by the calculation as in Table IV and Table V. Based on Table IV note if the data on post-test value is normal. This is known from the sig value Robomind Class in the Kolmogorov-Smirnov test of 0.095 and sig value Scratch Class in the Kolmogorov-Smirnov test of 0.200, the value is greater than the error rate (0.05). The Sig value in Levene test is 0,059 . The value is greater than the error rate (0.05), so it is known that the post-test results are homogeneous. The Sig (2-tailed) value in the t-test is 0.048 . The value is smaller than the error rate (0.05), so it is known that there is a significant difference between the two classes of post-test results.

\section{2. $\boldsymbol{R} \boldsymbol{Q}_{2}$ : Which one more effective between Robomind and Scratch?}

The mean values of the post-test results of both of sample groups were used to obtain the gain test. Based on Equatation 1, the value of gain that is less than zero/null indicaes that Robomind Class is more excellent than Scratch Class. However, the value of gain which is more than zero/null indicates that Scratch Class is more excellent than Robomind Class.

$$
\begin{gathered}
\langle g\rangle=\frac{\left(\%<S_{f}>-\%<S_{i}>\right)}{\left(S_{M 1}-\%<S_{i}>\right)}=\frac{(66,7411-61,4583)}{(100-61,4583)}=\frac{(5,2828)}{(38,5417)}=0,137 \\
|<g>|=|0,137|=0,137
\end{gathered}
$$

The result of gain value is 0.137 . Due to the acquisition of the gain value greater than 0 (positive value), it is concluded that the group with treatment in the form of Scratch use has better ability in understanding the basic concept of programming. In other words, the use of Scratch is more effective in enhancing the understanding of basic concepts of programming. Value $|\langle\mathrm{g}\rangle|$ Is 0.137 . The value is less than 0.3 so it can be rated as a $g$-low. In other words, Scratch is slightly more effective in improving the understanding of the basic concept of programming than Robomind.

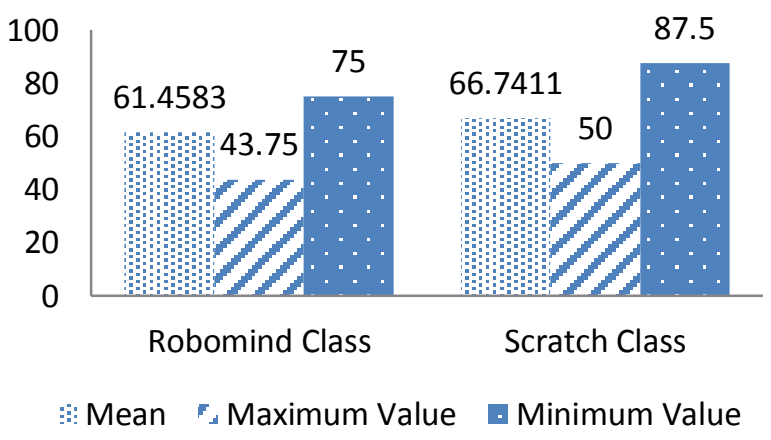

Figure 1. The Comparison Among Mean, Maximum, Minimum on The Post-Test Result 
Figure 1 shows that the mean of Scratch Class is more excellent than Robomind Class. Scratch Class also has the maximum value/score in which its minimum value/score is better than Robomind Class.

3. $\boldsymbol{R Q}_{3}$ : What are the aspects that differentiate Robomind and Scratch in improving understanding of the basic concept of programming?

\subsection{Analysis on Post-Test Result Based on Test Item Categories}

TABLE VI. COMPARISON GAIN IN EACH CATEGORY OF QUESTIONS

\begin{tabular}{|ccccc|}
\hline \multirow{2}{*}{ Gain } & \multicolumn{5}{c|}{ Category } \\
\cline { 2 - 5 } & $\mathrm{A}$ & $\mathrm{B}$ & $\mathrm{C}$ & $\mathrm{D}$ \\
\hline Value & 0.11 & 0.159 & 0.154 & 0.11 \\
Status & $g$-low & $g$-low & $g$-low & $g$-low \\
\hline
\end{tabular}

The result of post-test analysis using the gain test in each category of questions can be seen in Table VI. Category A is the ratio of the ability of learners in completing the decision making items, Category B is the ratio of the ability of learners in completing the looping item, Category $\mathrm{C}$ is the ratio of participants ability of learners in completing the test item accompanied by the programming language, and Category $\mathrm{D}$ is a comparison of the ability of learners in completing the question item without the accompanying programming languages. Based on Table VI it is known that students given Scratch are slightly superior (with a low gain value) in all categories of the test item.

\subsection{Early Introduction to PAT}

At this initial meeting, the two sample groups were divided into two based on the PAT used, namely Robomind Class (26 people) and Scratch Class (30 people). Both of groups were given the same task, namely to apply (explore) the concept of decision making and looping at each PAT maximum for 45 minutes with 45 minutes previously been given learning materials. The learning method used in this meeting is discovery learning.

The individuals who can implement branches in the PAT used denoted by 'decision making' and individuals who can only implement branches in the PAT used denoted by 'decision making only'. Individuals who can implement loops on the PAT used denoted by 'looping only' and individuals who can only implement loops on the PAT used denoted by 'looping'. The individual who can implement decision making and loop on the PAT used denoted by 'double' and whereas individuals who cannot implement both decision making and loop on the PAT used denoted by 'unable'.

In Table VII shows a large comparison of the gain value of the implementation of the basic concept of programming to the two classes. This comparison is done to find out the difference between the two classes in implementing the basic concept of programming in each PAT. In Table VII it is known that: (1) the number of doubleskilled individuals in the Robomind Class is superior to the very small differences; (2) The Robomind Class is superior in the decision making implementation abilities of the 
PAT over the Scratch Class; (3) The Scratch Class is very superior and very different higher than the Robomind Class in looping implementation abilities on PAT; (4) The Scratch Class is superior to the Robomind Class in the decision making or looping implementation abilities of the PAT.

TABLE VII. COMPARISON OF GAIN BASED ON CAPABILITIES IN THE EARLY INTRODUCTION OF PAT

\begin{tabular}{|ccccc}
\hline & \multicolumn{3}{c}{ Ability } & \\
\cline { 2 - 4 } Gain & Decision & Looping & Double & \\
& making & & & \\
\hline Value & $-0,37$ & 0,897 & $-0,055$ & $-0,382$ \\
Status & $g$-medium & $g$-high & $g$-low & $g$-medium \\
\hline
\end{tabular}

Note: The negative sign in the value indicates Robomind Class is superior to Scratch Class, and vice versa

In both classes (Robomind Class and Scratch Class) each class was assigned to implement the two basic concepts of programming on each PAT. The comparison of gain values in both capabilities in each PAT can be seen in Table VIII. This comparison is done to find out the major inequality of application of the basic concept of programming on each PAT. Based on the large differences in the capability of the two sample groups, it is known that Robomind's use has a better ability in terms of evenness of decision making and repetition implementation capabilities in PAT. This is because the value of the gain (difference in ability) owned is much smaller compared to the Scratch Class.

TABLE VIII. THE COMPARISON OF GAIN VALUES IN BOTH CAPABILITIES IN EACH PAT

\begin{tabular}{|ccc|}
\hline \multirow{2}{*}{ Gain } & \multicolumn{2}{c|}{ Class } \\
\cline { 2 - 3 } & Robomind & Scratch \\
\hline Value & $-0,293$ & 0,908 \\
Status & $g$-low & $g$-high \\
\hline
\end{tabular}

Note: The negative sign in the value indicates decision making capabilities is superior to looping capabilities, and vice versa

Comparison of the ability to implement the two basic concepts of programming classified by the gender of the learners can be seen in Table IX. From the calculation of gain value is known that: (1) the group of women is better in generating the number of people who are able to implement the two basic concepts of programming on PAT; (2) the female group is superior in the low (small) gap compared to the male group in implementing decision making capabilities in PAT; (3) the female group is superior to the male group in implementing the looping capability of the PAT. 
TABLE IX. COMPARISON OF THE ABILITY TO IMPLEMENT THE TWO BASIC CONCEPTS OF PROGRAMMING CLASSIFIED BY THE GENDER OF THE LEARNERS

\begin{tabular}{|ccccc|}
\hline & \multicolumn{3}{c}{ Ability } & \\
\cline { 2 - 4 } Gain & Decision & Looping & Double & \\
& making & & & \\
\hline Value & 0,295 & 0,339 & 0,41 & 0 \\
Status & $g$-low & g-low & $g$-low & Not different \\
\hline
\end{tabular}

Note: The negative sign in the value indicates the man is superior to women, and vice versa

\section{DISCUSSION}

The results of the test on post-test results using t-test are indicated if there is a significant difference from the use of each PAT in the ability to refine the basic concepts of programming concepts (consisting of the concept of decision making and looping). Thus, it can be said that the different concepts of environmental concepts about the impact of PAT on the difference in the ability to improve the understanding of basic concepts of programming in novice programmers. From the difference is known that the use of Scratch slightly superior by using the use of Robomind in improving the basic concept of programming. The conclusion is based on the gain test results. This is in line with Koorse's research when the use of the drag-and-drop interface makes Scratch does not apply syntax rules [13]. It aims to let users focus on the planning of the programming solution. Malan and Leitner [14] showed that there was a decrease in the number of learners who failed in learning the introduction of basic science computer science when Scratch used educators for the learning. Scratch is able to lead in developing the creativity of learners through innovative learning activities [15].

Based on the post-test result which categorized based on test item types: (1) the ability to resolve the decision making test items; (2) the ability to resolve the looping test items; (3) the ability to resolve the test items with the programming language (syntax); (4) the ability to resolve the test item without programming language (nonsyntax). from these result, it is known that the use of Scratch excel in all types of the test item with each category has a g-low rated gain value.

At the beginning of PAT introduction, learners required to implement both basic programming concepts on each PAT within 45 minutes. The results showed that the number of people who are able to implement the concept of decision making and looping (double capability) on Robomind usage is higher than Scratch usage, the use of Robomind is superior with the very little difference compared to the use of Scratch with a g-low rated gain value. Robomind's use is also superior in implementing decision making concepts compared to the use of Scratch with a g-medium rated gain value. However, in implementing the concept of looping the use of Scratch is far superior to the use of Robomind with a g-high rated gain value. In addition, the use of Scratch produces Robomind with a g-medium rated gain value. Based on the inability of the concepts of programming on each PAT, the use of Scratch has a much greater inequality than Robomind usage. The implementation of the looping concept on Scratch is much higher than the ability to implement decision making concepts. The ability to implement the concept of looping on the PAT that implements the system of instruction 
blocks (similar to Scratch) is capable of being applied by 68 people (43\% of The whole sample group) while the implementation ability of decision making concepts is only capable applied by 9 people (5.7\% of the overall sample group) [16]. The use of Scratch is able to significantly improve the people's creativity [17].

In the comparison of the abilities of gender-based, the following are the results: (1) both abilities of the implementation of the basic concept of decision making and the repetition of women are superior to men; (2) women have a higher percentage of individuals who are superior to the case of multiple-ability individuals (able to implement both programming concepts in PAT), the gain values are medium; (3) both men and women have the same percentage in the number of people who are unable to implement both basic programming concepts. It is based on the researches showing that the women have the better values in design studio, the higher score on the creativity index and objective value compared to the men in which the differences are insignificant [18]. In the matter of abstract, the men tend to use the ability in abstract thinking compared to the women. The women have the good spatial visualization ability while elaborating. However, the men have more excellent general spatial ability and visual cognitive ability than the women. It is showed by the women's inability to give problem solving strategies that are accurate and effective. Other researches show that the women have higher general intelligence than those that the men have [19]. Therefore, the intelligence compensates the weaknesses better than the visual art compared to the men even though it is not significant [20].

\section{CONCLUSION}

Based on the results of the study that aims to answer the problem statement or research question and the decision on the hypothesis taken concluded that there are differences in understanding the basic concept of programming between when using Robomind and when using Scratch. The use of Scratch is more effective (low gain gap) in improving understanding of basic programming concepts than Robomind's usage.

Students given Scratch are superior (with relatively low gain) in all categories of given items: Looping, decision making, understanding programming logic with the syntax of programming languages, understanding programming logic without programming syntax.

In the first ninety minutes of the first time recognizing PAT along with the assignment of the implementation of the concept of looping and decision making in each PAT. Learners given Scratch is superior to learners given Robomind in terms of excellent superiority in the category of loop implementation, superior to the medium difference in the category of minimizing the number of people who are not able to implement the two basic concepts of programming. Learners given Robomind is superior to learners given Scratch in terms of superior profusely in categories maximizing the number of people who are able to implement both basic programming concepts, superior in medium differences in decision making implementation categories. In terms of comparing the application of the two basic concepts to each PAT, the both PATs have very high inequality, learners who are given Robomind gives much better in this case because it has a much lower inequality than the learners who are given Scratch. In the category of implementation achievement of the two basic concepts of programming by gender, women excels in the category of decision making 
and looping implementation and dual ability (able to implement both the basic concepts of programming on PAT). Meanwhile, there is no difference between men and women in terms of minimizing the number of people who are unable to implement both basic programming concepts.

\section{REFERENCES}

[1] Koorsse, Melisa. 2012. An Evaluation of Programming Assistance Tools to Support the Learning of IT Programming: A Case Study in South African Secondary Schools. South Africa: Nelson Mandela Metropolitan University.

[2] Wilson, C., Sudol, L., Stephenson, C., dan Stehlik, M. 2010. Running on empty: The failure to teach K-12 Computer Science in the digital age. Tech. Rep. ACM.

[3] Yuana, R. A., Faisal M., Pangestu, D. dan Putri Y.R.L. 2015. "Math thematic learning through the introduction of basic science-based programming games virtual robot for high school students". Advanced Science Letters, 21(7): 2235-2238.

[4] Pullan, A., Drew S., dan Tucker S. 2013. "A Problem Based Approach to Teaching Programming". Proceedings of the International Conference on Frontiers in Education: Computer Science and Computer Engineering (FECS), 1(6): 1-6.

[5] Pitakasari, Ajeng Ritzki. 2013. "Indonesia Miliki Potensi Pasok Pakar Software Dunia". [Online]. Available: www.republika.co.id. [Accessed: 18-Jan-2017]

[6] Csikszentmihalyi, Mihaly. 1998. Finding Flow; The Psychology of Engangement with Everyday Life. Basic Books.

[7] Pears, A., Seidman, S., Malmi, L., Mannila, L., Adams, E., Bennedsen, J., et al. 2007. "A survey of literature on the teaching of introductory programming". In working group reports on ITiCSE on Innovation and technology in computer science education, ITiCSE-WGR '07, pp. 204-223.

[8] Yuana, R. A., Maryono, D. 2016. "Robomind Utilization to Improve Student Motivation and Concept in Learning Programming”. Proceedings of International Conference on Teacher Training and Education (ICTTE) 2015, 1(1): 962-966.

[9] Nofitasari, A., Yuana, R. A., Maryono, D. 2015. "The Use of Robomind Application in Problem Based Learning Model to Enhance Student's Understanding in the Conceptual Programming Algorithm". Indonesian Journal of Informatics Education, 1(1): 10-18.

[10] Krathwohl, D. R. 2002. A Revision of Bloom's Taxonomy: An Overview. College of Education, The Ohio State University.

[11] Koorsse, M., Cilliers, C., Calitz, A. 2015. "Programming assistance tools to support the learning of IT programming in South African secondary school". Computer and Education Journal, 82, 162-178.

[12] Budiyono. 2004. Statistika Dasar untuk Penelitian. FKIP UNS Press. Surakarta.

[13] Koorsse, M., Cilliers, C., Calitz, A. 2015. "Programming assistance tools to support the learning of IT programming in South African secondary school". Computer and Education Journal, 82(1): 162-178.

[14] Malan, D., \& Leitner, H. 2007. "Scratch for budding computer Scientists". Proceedings of the $38^{\text {th }}$ SIGCSE Technical Symposium on Computer Science Education (SIGCSE'07), pp. 223227.

[15] Kobsiripat, Worarit. 2014. "Effects of the media to promote the scratch programming capabilities creativity of elementary school students". Procedia - Social and Behavioral Sciences, 174(2015): 227-232. 
[16] Chao, P. Y. 2016. "Exploring students' computational practice, design and performance of problem-solving through a visual programming environment". Computers \& Education Journal, 95(1): 202-215.

[17] Bustillo, Jon, \& Garaziar, Pablo. 2016. "Using Scratch to foster creativity behind bars: Two positive experiences in jail”. Thinking Skills and Creativity Journal. 19(1): 60-72.

[18] Cho, Ji Young. 2017. "An investigation of design studio performance in relation to creativity, spatial ability, and visual cognitive style". Thinking Skills and Creativity, 23 (2017): 67-78.

[19] Ho, C. H. 2006. Spatial cognition in design. Georgia Institute of Technology, Atlanta.

[20] Caballero, A.O., Casares, J.A.V., dan Caballero, M. A. 2017. "Visual perception in art education. Gender and intercultural study". Procedia - Social and Behavioral Sciences, 237 ( 2017 ): $588-593$. 